\title{
Conocimientos y actitudes hacia el VIH/SIDA: diferencias entre adolescentes españoles con padres casados y divorciados
}

\author{
Elena Carratalá, ${ }^{1}$ José Pedro Espada, ${ }^{1}$ Mireia Orgilés ${ }^{1}$
}

Artículo original

\section{SUMMARY}

\section{Objective}

This study is the first to examine whether the level of knowledge and the attitudes that a Spanish-speaking sample of adolescents have towards HIV/AIDS, both variables traditionally associated with risky practices, differs in function of having married or divorced parents, in order to determine whether the family situation should be included in the prevention programs.

\section{Materials and Method}

An observational cross-sectional study was carried out in fifteen secondary schools from five Spanish counties. Participants were 342 adolescents aged between 14 and 17 years, $50 \%$ with divorced parents. Participants completed the HIV/AIDS Knowledge Scale on Spanish Adolescents and the Scale of Attitudes towards HIV/AIDS.

\section{Results}

Adolescents with divorced parents reported more favorable attitudes towards HIV/AIDS $(p<.01 ; d=0.32)$, condom use $(p<.05 ; d=0.24)$, conducting HIV tests $(p<.05 ; d=0.28)$, and people living with HIV $(p<.001 ; 0.32)$.

\section{Conclusions}

The results suggest that divorced parents could transmit to their children favorable attitudes towards AIDS and prevention methods. Including sessions with parents in the prevention programs is highlighted, aiming to encourage parents to transmit their adolescent children positive attitudes to prevent AIDS and other sexually transmitted diseases.

Key words: HIV, AIDS, adolescents, family structure.

\section{RESUMEN}

\section{Objetivo}

Examinar por primera vez con una muestra de habla hispana si existen diferencias entre los adolescentes con padres casados y divorciados en el nivel de conocimientos y en sus actitudes hacia el VIH/SIDA, variables tradicionalmente asociadas con las prácticas sexuales de riesgo, a fin de conocer si la situación familiar debe tenerse en cuenta en los programas de prevención.

\section{Material y método}

Se trata de un estudio transversal observacional, en el que participaron quince centros escolares de cinco provincias de España. Los participantes fueron 342 adolescentes de entre 14 y 17 años, el 50\% de ellos con padres divorciados. Completaron de forma anónima la Escala de Conocimientos sobre el VIH/SIDA para Adolescentes Españoles y el Cuestionario de Actitudes hacia el VIH/SIDA.

\section{Resultados}

Los adolescentes con padres divorciados mostraron actitudes más favorables hacia el $\mathrm{VIH} / \mathrm{SIDA}(p<.01 ; d=0.32)$, uso de preservativo $(p<.05$; $d=0.24)$, realización del test del $\mathrm{VIH}(p<.05 ; d=0.28)$, y hacia las personas que viven con el $\mathrm{VIH}(\mathrm{p}<.001 ; 0.32)$. No se hallaron diferencias significativas en el nivel de conocimientos en función de la estructura familiar.

\section{Conclusiones}

Los resultados sugieren que los padres divorciados transmiten a sus hijos una actitud más favorable hacia el uso del preservativo. Se recomienda incluir en los programas de prevención sesiones dirigidas a los padres, para fomentar la transmisión a los hijos de actitudes favorables al sexo seguro que les prevengan del SIDA y de otras enfermedades de transmisión sexual.

Palabras clave: VIH, SIDA, adolescentes, estructura familiar.

\section{INTRODUCCIÓN}

El VIH/SIDA continúa siendo un importante problema de salud pública, con 33.3 millones de personas en el mundo afectadas y 1.8 millones de defunciones en el último año registrado. Anualmente se producen más de 7000 nuevas infecciones, el $41 \%$ en jóvenes con edades comprendidas entre 15 y 24 años. ${ }^{1}$ Entre el 75\% y el 80\% de las infecciones por VIH ocurren como consecuencia de una relación sexual no protegida, y un tercio de los casos tiene lugar al mante-

Departamento de Psicología de la Salud. Universidad Miguel Hernández de Elche. España.

Correspondencia: Elena Carratalá. Departamento de Psicología de la Salud, Universidad Miguel Hernández. Av. de la Universidad s/n, 03202 Elche, Alicante, España. Tel: (+96) 665 - 8348. E-mail: morgiles@umh.es

Recibido primera versión: 8 de octubre de 2012. Segunda versión: 12 de marzo de 2013. Aceptado: 23 de mayo de 2013. 
ner relaciones heterosexuales. ${ }^{2}$ A pesar del aumento de los programas de prevención, los jóvenes continúan llevando a cabo conductas sexuales de riesgo, ${ }^{3}$ lo que hace necesario llevar a cabo investigaciones que mejoren su eficacia. Pese a que los últimos datos indican un descenso debido a la eficacia de los tratamientos antirretrovirales, España sigue siendo uno de los países de Europa Occidental con mayor incidencia de SIDA, con una estimación de 1162 nuevos casos diagnosticados en el año $2010{ }^{4}$

Los modelos teóricos que explican las conductas sexuales de riesgo prestan especial atención al papel de los factores cognitivos y motivacionales como precursores o determinantes del comportamiento. ${ }^{5}$ Entre las variables más relacionadas con las prácticas sexuales de riesgo destaca el nivel de información sobre las conductas de riesgo y sobre los métodos de protección. ${ }^{6}$ Numerosos estudios evidencian el desconocimiento de los adolescentes hacia el VIH/SIDA y hacia las medidas que pueden llevar a cabo para prevenir la infección. ${ }^{6-8}$ Aumentar el nivel de conocimientos de los adolescentes hacia el VIH es una estrategia que ha demostrado su eficacia en los programas de prevención, ${ }^{9}$ pero, pese a que se considera importante, un nivel de conocimiento adecuado sobre el VHI/SIDA no es suficiente para llevar a cabo conductas preventivas. ${ }^{10}$ Junto al nivel de información, los modelos teóricos sobre conductas de salud consideran que las actitudes hacia las conductas de protección y hacia el VIH/SIDA son una variable importante que influye en los comportamientos de riesgo. ${ }^{11}$ La falta de conocimientos e información se relaciona de forma directa con una actitud negativa hacia los métodos de protección de las prácticas de riesgo, quedando por tanto expuestos a la infección. ${ }^{12}$ Ambas variables, conocimientos y actitudes sobre el SIDA, condicionan la práctica de conductas sexuales saludables, ${ }^{9}$ por lo que su estudio de forma conjunta es importante como factor mediador de las conductas sexuales de riesgo en los adolescentes.

En el desarrollo sexual de los adolescentes la familia tiene un papel fundamental como principal agente de socialización de los hijos. Durante la adolescencia se produce un incremento de la inestabilidad emocional, la conflictividad y las conductas de riesgo. ${ }^{13}$ La vulnerabilidad del adolescente puede aumentar como consecuencia de determinados cambios en la situación familiar, como el divorcio de los padres, que se ha relacionado con diversas conductas problemáticas en los hijos, entre ellas la práctica de conductas sexuales de riesgo. ${ }^{14,15} \mathrm{Al}$ comparar la conducta sexual de los adolescentes con padres casados y divorciados, trabajos previos a nivel internacional han hallado en estos últimos una mayor probabilidad de tener relaciones sexuales, ${ }^{15,16}$ mayor frecuencia en la actividad sexual, ${ }^{17}$ un mayor número de parejas sexuales, ${ }^{18}$ una edad más temprana del inicio sexual, ${ }^{19,20}$ y más riesgo de embarazo. ${ }^{21}$

Hasta el momento, los estudios que se han llevado a cabo evidencian las diferencias en las conductas sexuales de los adolescentes en función de la estructura familiar, pero ningún trabajo ha examinado si difieren los conocimientos y actitudes que poseen hacia las prácticas sexuales de riesgo. El objetivo del presente estudio es por tanto conocer si los conocimientos y las actitudes que tienen los adolescentes hacia el VIH/SIDA y hacia las prácticas sexuales de riesgo se relacionan con la estructura familiar (tener padres casados o padres divorciados), de modo que puedan diseñarse programas de prevención más ajustados a las necesidades de los jóvenes en función de su situación familiar. En base a los estudios previos que informan de más variables de riesgo cuando los padres están divorciados, se hipotetiza que los adolescentes cuyos padres se han divorciado presentarán un menor nivel de conocimientos y una actitud menos favorable hacia el uso del preservativo y hacia otros aspectos relacionados con la sexualidad y el VIH/SIDA.

\section{MATERIAL Y MÉTODOS}

\section{Participantes}

Se trata de un estudio observacional transversal. Para el reclutamiento de la población de estudio se estableció contacto, en base a su disponibilidad, con 15 colegios públicos y concertados de cinco provincias de España. Tres colegios estaban ubicados en el norte del país, tres en el sur, dos en el este, cuatro en el sudeste y tres en la zona central. Nueve se seleccionaron de la costa y los otros seis del interior. Se incluyeron colegios procedentes de zonas rurales y urbanas. Se proporcionó información a los directores de los centros y se solicitaron los permisos necesarios. Previamente, un comité de ética de la investigación experimental con sujetos humanos aprobó el estudio.

Se evaluó a todos los adolescentes con edades comprendidas entre 14 y 17 años cuyos padres proporcionaron consentimiento informado escrito para que sus hijos participaran en la investigación ( $n=1.216 ; 97 \%$ del total de estudiantes). A continuación fueron seleccionados del total de la muestra los sujetos que procedían de familias con padres divorciados $(n=171)$. Para comparar el grado de conocimientos y las actitudes en función de la estructura familiar, se seleccionó al azar una submuestra de adolescentes de la muestra total con las mismas características de edad, sexo, colegio y nivel socioeconómico, pero con padres casados. La muestra final estaba formada por 342 adolescentes de edades comprendidas entre 14 y 17 años $(M=15,85 ; D T=.77)$, de raza caucásica, de los que el 39\% eran varones, y un nivel socioeconómico medio, determinado en función de la ubicación de los centros escolares y de la actividad laboral de los padres.

Para la obtención de los datos los participantes respondieron los cuestionarios en clase en grupos de 20 estudiantes aproximadamente. Se leyeron las instrucciones en voz alta contestando a todas las dudas que surgieron. Los cuestiona- 
rios eran totalmente anónimos y se contrabalancearon, de manera que cada estudiante recibió el cuestionario en un orden diferente al de los estudiantes que tenía a su alrededor. Para garantizar la privacidad de las respuestas, se proporcionó un sobre a cada uno de los participantes para guardar el cuestionario una vez completado. Ningún participante dejó menos de un $20 \%$ de ítems sin responder, de modo que ningún caso fue excluido del análisis estadístico.

\section{Mediciones principales}

Para la evaluación de los conocimientos sobre las prácticas de riesgo, se administró la Escala de Conocimientos sobre el VIH/SIDA para adolescentes españoles (HIV-KS). ${ }^{9}$ Está compuesta por 10 ítems distribuidos en tres subescalas: transmisión oral del VIH, efectos del VIH y otras vías de transmisión del VIH. El primer factor mide los conocimientos sobre comportamientos de riesgo donde hay contacto con la saliva. El segundo factor examina los conocimientos básicos sobre aspectos biológicos de la infección y el tercer factor evalúa la información sobre las principales vías de transmisión del VIH. Las propiedades psicométricas de la escala son adecuadas, con una consistencia interna de .71 para la escala total, .78 para la subescala transmisión oral, .57 para efectos del VIH y .61 para otras vías de transmisión. ${ }^{9}$

Para la evaluación de las actitudes hacia las prácticas de riesgo, se administró la Escala de Actitudes hacia el VIH/SIDA (HIV-AS), ${ }^{22}$ formada por 12 ítems, clasificados en cuatro factores. El primer factor mide la actitud hacia el sexo seguro cuando existen obstáculos, que hace referencia a la percepción del sujeto en relación con hacer frente a situaciones en que el comportamiento seguro depende de ser asertivo. El segundo factor evalúa las actitudes hacia la prueba del VIH, e incluye las situaciones de realizarse o recomendar la prueba del VIH a otras personas después de un comportamiento de riesgo. El tercer factor examina las actitudes hacia el uso del preservativo, y el cuarto factor las actitudes hacia las personas que viven con el síndrome e incluye las situaciones de apoyo, el malestar y la relación cercana con las personas afectadas. La HIV-AS presenta una consistencia interna adecuada tanto para el total de la escala $(\alpha=.81)$ como para las subescalas ( $\alpha=.76$ para la actitud hacia el sexo seguro cuando existen obstáculos, $\alpha=.75$ para la actitud hacia la prueba del VIH, $\alpha=.70$ para la actitud hacia el uso del preservativo, y $\alpha=.69$ para la actitud hacia las personas afectadas).22

\section{Análisis estadístico}

Las diferencias en conocimientos y actitudes hacia el VIH/ SIDA y las prácticas de riesgo entre los adolescentes con padres divorciados y con padres casados fueron examinadas mediante la prueba $t$ de Student para dos muestras independientes con el programa estadístico PASW. Se calcularon los tamaños del efecto mediante la diferencia de medias tipificada para conocer la magnitud de las diferencias, considerándose 0.20 una magnitud del efecto pequeña, 0.50 mediana y 0.80 grande. $^{23}$

\section{RESULTADOS}

El cuadro 1 muestra la comparación entre los adolescentes con padres divorciados y con padres casados en las variables de conocimientos y actitudes hacia las prácticas de riesgo. El nivel de conocimiento sobre el VIH/SIDA fue similar en ambos tipos de estructura familiar. Los sujetos con padres divorciados presentaron actitudes más favorables hacia la realización del test del VIH $(p<.05)$, el uso del preservativo $(p<.05)$ y las personas con VIH $(p<.001)$. El tamaño del efecto resultó pequeño en las tres comparaciones $(d=0.26, d=0.24$, $d=.35$; respectivamente).

Cuadro 1. Conocimientos y actitudes hacia el VIH/SIDA

\begin{tabular}{|c|c|c|c|c|c|c|}
\hline & \multicolumn{2}{|c|}{$\begin{array}{l}\text { Divorciados } \\
(n=171)\end{array}$} & \multicolumn{2}{|c|}{$\begin{array}{l}\text { No divorciados } \\
\qquad(\mathrm{n}=171)\end{array}$} & \multirow[b]{2}{*}{$t$} & \multirow[b]{2}{*}{$d$} \\
\hline & $M$ & $\mathrm{DE}$ & $M$ & $\mathrm{DE}$ & & \\
\hline \multicolumn{7}{|l|}{ Conocimientos sobre el VIH/SIDA } \\
\hline - Transmisión oral VIH & 1.42 & 1.26 & 1.40 & 1.21 & -0.13 & - \\
\hline - Efectos VIH & 0.36 & 0.70 & 0.28 & 0.64 & -1.13 & - \\
\hline - Otras vías de transmisión VIH & 3.39 & 0.89 & 3.24 & 1.09 & -1.35 & - \\
\hline - Total & 5.16 & 1.96 & 4.94 & 2.12 & -1.03 & - \\
\hline \multicolumn{7}{|l|}{ Actitudes hacia el VIH/SIDA } \\
\hline - Obstáculos & 9.78 & 2.01 & 9.73 & 2.03 & -0.27 & - \\
\hline - Test VIH & 7.08 & 1.22 & 6.76 & 1.27 & $-2.39 *$ & 0.26 \\
\hline - Uso preservativo & 14.22 & 1.86 & 13.76 & 2.04 & $-2.16^{*}$ & 0.24 \\
\hline - Personas VIH & 9.76 & 1.82 & 9.08 & 2.04 & $3.25^{* * *}$ & 0.35 \\
\hline - Total & 40.84 & 4.58 & 39.33 & 4.74 & 3.01 ** & 0.32 \\
\hline
\end{tabular}




\section{DISCUSIÓN}

En el presente estudio se examina el nivel de conocimientos y las actitudes hacia el VIH/SIDA y las prácticas sexuales de riesgo en un grupo de adolescentes de habla hispana con padres casados y con padres divorciados para examinar si la estructura familiar se relaciona con estas variables. Estudios anteriores han puesto de manifiesto que las conductas sexuales de los adolescentes con padres divorciados difieren de las de aquéllos que tienen padres casados. ${ }^{16,18,20}$ Sin embargo, a pesar de la alta frecuencia de las rupturas de pareja en España, siendo el país de la Unión Europea con el mayor incremento del número de divorcios en la última década, ${ }^{24}$ y de la relación entre las conductas sexuales y la estructura familiar, ningún estudio había examinado hasta el momento los conocimientos y actitudes sobre el VIH/SIDA de los adolescentes con padres divorciados en comparación con los de los adolescentes con padres casados.

Los resultados de nuestro estudio indican, contrariamente a lo esperado, que el nivel de conocimientos sobre el VIH/SIDA no parece relacionarse con la estructura familiar, ya que no difiere entre los adolescentes con padres casados y con padres divorciados. Los resultados sugieren que la educación sexual que proporcionan los padres, con independencia de la situación familiar, tiene efectos semejantes en los adolescentes a nivel cognitivo. La ausencia de diferencias en el nivel de conocimiento sobre el VIH/SIDA en función de la estructura familiar podría explicarse atendiendo a las fuentes de transmisión de la información. Algunos estudios afirman que los adolescentes obtienen la información sobre la sexualidad y los métodos de prevención del VIH y de otras enfermedades de transmisión sexual principalmente del contexto escolar y de los amigos. ${ }^{6,12} \mathrm{El}$ hecho de que sea la escuela o los amigos, y no los padres, los que proporcionan información sobre el VIH/SIDA podría explicar que el nivel de conocimientos de los adolescentes sea el mismo con independencia de si los padres están casados o divorciados.

En la segunda variable examinada, las actitudes hacia el síndrome y las prácticas de riesgo, se hallaron diferencias significativas en función de la estructura familiar. En este sentido, y en contra de nuestra hipótesis, son los hijos de padres divorciados los que mantienen una actitud más favorable hacia el uso del preservativo, hacia la realización del test del VIH y hacia las personas con VIH/SIDA. Dicha actitud más favorable hacia el sexo seguro en los adolescentes con padres divorciados podría explicarse desde la teoría del aprendizaje social, que indica que las parejas divorciadas se muestran más permisivas y manifiestan más aceptación de las relaciones premaritales de los hijos. ${ }^{25}$ La mayor aprobación de los padres divorciados de las relaciones sexuales de sus hijos adolescentes podría llevarles a aplicar estrategias más activas para fomentar en ellos conductas sexuales saludables, mediante la transmisión de actitudes más positivas hacia las relaciones sexuales protegidas y hacia la infección por VIH.

En resumen, los resultados indican que los adolescentes con padres divorciados tienen actitudes más favorables hacia el sexo seguro y hacia el SIDA, pero su nivel de conocimientos no difiere de los adolescentes con padres casados. Pese a que estudios previos a nivel internacional informan de más variables de riesgo en los adolescentes con padres divorciados, los resultados del presente estudio sugieren que los adolescentes españoles cuyos padres están divorciados no presentan más riesgo frente a las conductas sexuales e infección por el VIH en cuanto a conocimientos y actitudes se refiere. Por el contrario, presentan una actitud más favorable hacia las relaciones sexuales protegidas. Como principal aportación, el estudio aumenta el conocimiento científico sobre la prevención de conductas sexuales de riesgo en los adolescentes, examinando por primera vez con una muestra española el nivel de conocimientos y las actitudes hacia el VIH/SIDA y las prácticas de riesgo en relación con la estructura familiar.

El presente estudio presenta ciertas limitaciones. La principal es que se trata de un estudio transversal, lo que puede afectar a la interpretación de los resultados y sugiere la necesidad de llevar a cabo estudios longitudinales. Además, sería interesante en estudios futuros profundizar sobre el tipo de actitudes que transmiten los padres a sus hijos, examinando si, como se sugiere en nuestro estudio, éstas difieren en función de la estructura familiar. Del mismo modo, sería conveniente conocer determinadas características de la interacción familiar como la accesibilidad y disponibilidad informativa de los padres.

La aplicabilidad práctica de los resultados es clara. Los resultados resaltan la necesidad de atender a variables familiares en la implementación de los programas de prevención de las conductas sexuales de riesgo de los adolescentes. Las diferencias en las actitudes hacia las prácticas de riesgo y el SIDA en función de la estructura familiar sugieren la importancia de los padres como transmisores de actitudes a sus hijos. Los adolescentes con padres divorciados probablemente transmitan a sus hijos adolescentes actitudes más favorables hacia el sexo seguro lo que constituye una importante variable de protección frente a ésta y otras enfermedades de transmisión sexual. Diseñar programas de prevención en los que se incluyan sesiones dirigidas a los padres podría contribuir a la transmisión de padres a hijos de actitudes que favorezcan su prevención temprana.

\section{REFERENCIAS}

1. Informe de ONUSIDA sobre la epidemia mundial de SIDA 2010. Descargado el 22 de enero de 2011 de http://www.unaids.org/

2. De Bedout A. El VIH/SIDA: Factores psicosociales y comportamentales. Rev Facultad Ciencias Salud 2008;5:129-140.

3. Piña JA. Eventos disposicionales que probabilizan la práctica de conductas de riesgo para el VIH/SIDA. Anales Psicología 2004;20:23-32. 
4. Vigilancia epidemiológica del SIDA en España. Situación a 30 de junio de 2011. Ministerio de Sanidad, Política Social e Igualdad. Descargado de: http://www.msc.es/novedades/docs/InformeVIH-sida_junio2011.pdf

5. Fisher JD, Fisher WA. Theoretical approaches to individual-level change in VIH risk behaviour. En: Handbook of HIV prevention. Peterson JL, DiClemente RJ (eds.). New York: Kluwer-Plenum Press; 2000; pp. 3-56.

6. Macchi ML, Benítez S, Corvalán A, Núñez C et al. Conocimientos, actitudes y prácticas acerca del VIH/SIDA en jóvenes de nivel medio de Educación, del área metropolitana, Paraguay. Revista Chilena Pediatría. 2008;79:206-217.

7. Faílde JM, Lameiras M, Bimbela JL. Prácticas sexuales de chicos y chicas españoles de 14-24 años de edad. Gaceta Sanitaria 2008;22:511-519.

8. Rivas E, Rivas A, Barría R, Sepúlveda C. Conocimientos y actitudes sobre VIH/SIDA de estudiantes de enfermería de las Universidades de la frontera y austral de Chile. Temuco-Valdivia, Chile, 2004. Ciencia y enfermería. 2009;15:109-119.

9. Espada JP, Huedo-Medina T, Orgilés M, Secades R et al. Psychometric properties of the HIV/AIDS Knowledge Scale for Spanish adolescents (HIV-KS). Health Addictions 2009;9:149-164.

10. Díaz-Loving R. Una visión integral de la lucha contra el VIH-SIDA. Revista Interamericana Psicología 2001;35:25-39.

11. Sánchez F. Representaciones psicosociales sobre el SIDA. Revista Información Psicológica 1997;63:198-200.

12. Santín C, Torrico E, López MJ, Revilla C. Conocimiento y utilización de los métodos anticonceptivos y su relación con la prevención de enfermedades de transmisión sexual en jóvenes. Anales Psicología 2003;19:81-90.

13. Arnett JJ. Adolescent storm and stress, reconsidered. American Psychologist. 1999;54:317-326.

14. Lansford JE. Parental divorce and children's adjustment. Perspectives Psychological Science 2009;4:140-152.

15. Cavanagh SE, Crissey SR, Raley RK. Family structure history and adolescent romance. J Marriage Family 2008;70:698-714.
16. Donahue KL, D’Onofrio BM, Bates JE, Lansford JE et al. Early exposure to parents' relationship instability: implications for sexual behaviour and depression in adolescence. J Adolescent Health 2010:47:547-554.

17. Ellis BJ, Bates JE, Dodge KA, Fergusson DM et al. Does father absence place daughters at special risk for early sexual activity and teenage pregnancy? Child Development 2003;74:801-821.

18. Cleveland $\mathbf{H H}$, Gilson $\mathrm{M}$. The increased importance of mother-child relationships on sexual behaviors of adolescents in disadvantaged neighborhoods. J Youth Adolescence 2004;33:319-329.

19. D'Onofrio BM, Turkheimer EN, Emery RE, Slutske $W$ et al. A genetically informed study of the processes underlying the association between parental marital instability and offspring adjustment. Developmental Psychology 2006;42:486-499.

20. Zimmer-Gembeck MJ, Helfand $M$. Ten years of longitudinal research on U.S. adolescent sexual behavior: The evidence for multiple pathways to sexual intercourse, and the importance of age, gender and ethnic background. Developmental Review 2008;28:153-224.

21. Hogan DP, Sun RJ, Cornwell GT. Sexual and fertility behaviours of American females aged 15-19 years: 1985, 1990, and 1995. American J Public Health 2000;90:1421-1425.

22. Espada JP, Ballester R, Huedo-Medina T, Secades-Villa R et al. Development of a new instrument to assess AIDS-related attitudes among Spanish Youngsters. Anales Psicología 2013;29:83-89.

23. Cohen J. Statistical power analysis for the behaviorla sciences. Segunda edición. Hillsdale, NJ: Lawrence Erlbaum Associates, Publishers; 1988.

24. Informe Evolución de la Familia en España 2010. Instituto de Política Familiar. Descargado el 27 de julio de 2012 de http://www.ipfe.org/documentacion.htm.

25. Davis CE, Friel L. Adolescent sexuality: Disentangling the effects of family structure and family context. J Marriage Family 2001;63:669-681.

Artículo sin conflicto de intereses 